\title{
Exact Reconstruction From Uniformly Attenuated Helical Cone-Beam Projections in SPECT
}

Qiu Huang, Jiangsheng You, Gengsheng L. Zeng, and Grant T. Gullberg

E. O. Lawrence Berkeley National Laboratory,

Life Science Division

Berkeley, CA 


\begin{abstract}
In recent years the development of cone-beam reconstruction algorithms has been an active research area in $x$-ray computed tomography (CT), and significant progress has been made in the advancement of algorithms. Theoretically exact and computationally efficient analytical algorithms can be found in the literature. However, in single photon emission computed tomography (SPECT), published cone-beam reconstruction algorithms are either approximate or involve iterative methods. The SPECT reconstruction problem is more complicated due to degradations in the imaging detection process, one of which is the effect of attenuation of gamma ray photons. Attenuation should be compensated for to obtain quantitative results. In this paper, an analytical reconstruction algorithm for uniformly attenuated cone-beam projection data is presented for SPECT imaging. The algorithm adopts the DBH method, a procedure consisting of differentiation and backprojection followed by a finite inverse cosh-weighted Hilbert transform. The significance of the proposed approach is that a selected region of interest can be reconstructed even with a detector with a reduced field of view. The algorithm is designed for a general trajectory. However, to validate the algorithm, a numerical study was performed using a helical trajectory. The implementation is efficient and the simulation result is promising.
\end{abstract}

Index Terms: cone-beam, SPECT, attenuation correction.

Qiu Huang is with the E. O. Lawrence Berkeley National Laboratory, One Cyclotron Road, Mail Stop 55R0121, Berkeley, CA 94720, USA. Telephone: (510) 495-2714. E-mail: QHuang@lbl.gov

Jiangsheng You is with the Cubic Imaging LLC, 264 Grove St., Auburndale, MA 02466, USA. E-mail: JYou@cubic-imaging.com

Gengsheng L. Zeng is with the Utah Center for Advanced Imaging Research (UCAIR), Department of Radiology, University of Utah, 729 Arapeen Drive, Salt Lake City, UT 84108, USA. Telephone: (801) 581-3918. E-mail: Larry@ucair.med.utah.edu

Grant T. Gullberg is with the E. O. Lawrence Berkeley National Laboratory, One Cyclotron Road, Mail Stop 55R0121, Berkeley, CA 94720, USA. Telephone: (510) 486-4768. E-mail: GTGullberg@lbl.gov 


\section{INTRODUCTION}

Much work has been accomplished in the development of analytical cone-beam reconstruction algorithms in three dimensional (3D) x-ray computed tomography (CT). Early accomplishments include the efficient yet approximate Feldkamp-Davis-Kress reconstruction algorithm [1], a theorem for data sufficiency conditions [2], an exact inversion reconstruction based on the 3D Fourier transform [2], and exact algorithms based on the 3D Radon transform [3], [4]. Recently, Katsevich made a significant contribution with the development of a filtered backprojection algorithm [5]. A backprojection of differentiated (DBP) projections and finite Hilbert transform approach, the DBH method, was then developed [6] as an improvement to Katsevich's method. The DBH method addresses the long object problem and is able to reconstruct any selected region of interest (ROI) from minimum data [6]. Unfortunately, these significant achievements cannot be easily adapted to the cone-beam problem in SPECT since gamma ray photons are attenuated before they are captured by the detector. In cone-beam SPECT, the most representative works in image reconstruction with attenuation compensation are inefficient iterative algorithms [7]-[10] and approximate analytical algorithms [11]-[14]. With the greater computing power that is now available, iterative methods are the algorithms of choice because they model the physics of the imaging detection process better and provide more accurate reconstructions. However, newly developed analytical algorithms lead to new insights into the mathematics of computerized tomography in SPECT, and new insights are important in the development, evaluation and understanding of iterative reconstruction algorithms. In this paper, a theoretically exact analytical algorithm is proposed and validated by a numerical study.

The algorithm presented here is a counterpart to the DBH method for CT reconstruction [6]. Due to the attenuation effect, the Hilbert transform in the CT reconstruction becomes the cosh-weighted Hilbert transform. The idea of reconstructing images with differentiation and backprojection followed by a finite inverse cosh-weighted Hilbert transform is an interesting topic in two-dimensional (2D) SPECT image reconstruction [15]-[19]. Theories of the finite inverse cosh-weighted Hilbert transform such as the existence, uniqueness, and stability of the inversion (which also theoretically support this work) were addressed in [15], [17], [18]. The DBH in 2D yields, theoretically, reconstruction of ROIs from uniformly attenuated data acquired over a range less than $2 \pi$, even with a relatively

small detector. The extension of the $\mathrm{DBH}$ method to $3 \mathrm{D}$ applications preserves the virtues of the method. In the helical cone-beam geometry, the DBH method has an intrinsic ability to solve both the long object problem and the truncation problem.

The proposed algorithm compensates for the attenuation effect to achieve a quantitatively accurate image from the uniformly attenuated cone-beam data. In SPECT, the correction for uniform attenuation is applicable to brain imaging [20]. The DBH method also makes feasible the reconstruction of an ROI from truncated projections, which is a helpful quality when performing anterior scanning close to the patient using a small detector plane. The 
algorithm is presented for a general cone-beam geometry, and thus is suitable for scans on a helical trajectory with a cone-beam collimator or a pinhole collimator.

The paper is organized as follows: First, we present the DBH method for a general cone-beam trajectory. Section II shows how the backprojection of differentiated projections of cone-beam projection data is related to the distribution of the radioactive tracer in SPECT. Section III presents how the finite inversion of the cosh-weighted Hilbert transform can restore the distribution function. Second, to validate the algorithm, we choose the helical cone-beam trajectory, since a helical cone-beam scan can readily be performed with existing SPECT systems by translating the bed while rotating cone-beam collimated detectors. The numerical study using a modified Shepp-Logan phantom is presented in Section IV. Finally, our conclusion is presented in Section V.

\section{DBP Method For Cone-Beam Projection Data}

In SPECT, the image to be reconstructed is the distribution of a radioactive tracer inside the patient's body, denoted by a smooth function $f(\vec{r})$ defined on a convex support $\Omega$. Without loss of generality, $\vec{r}=(x, y, z) \in \Omega=$ $\left\{(x, y, z): x^{2}+y^{2}<R_{0}^{2}\right\}$. Using a cone-beam collimator, for a fixed position of the detector, all emissions detected converge to one focal point $A$, see Fig. 1. The moving orbit of the focal point $A$ in a $3 \mathrm{D}$ space can be parameterized by $\lambda$. The vector pointing to the point $A$ from the origin is denoted by $\vec{a}(\lambda)$, which is a parameterization of the focal point throughout the paper. Then the measured projection data can be converted to the exponential ray transform of $f(\vec{r})$ :

$$
p(\lambda, \vec{\alpha})=\int_{0}^{\infty} d t e^{\mu t} f(\vec{a}(\lambda)+t \vec{\alpha})
$$

where $\mu$ is the linear attenuation coefficient and $\vec{\alpha}$ is a unit vector along the emission direction of the gamma ray photons. Image reconstruction in this work involves obtaining $f(\vec{r})$ from projections passing through $\vec{r}$, assuming a priori information about $\mu$ is available. From here forward, we use projections to indicate the exponential ray transform of $f(\vec{r})$, since the two sets of data are equivalent as a result of one-to-one mapping.

At some focal point with parameter $\lambda$, the direction of emissions passing through the point $\vec{r}$ is denoted by $\vec{\alpha}^{*}=\frac{\vec{r}-\vec{a}(\lambda)}{\|\vec{r}-\vec{a}(\lambda)\|}$. The DBP of projections passing through $\vec{r}$ has the property presented in the following theorem.

Theorem 1: Let $f(\vec{r})$ be a function with convex support. The projection data $p(\lambda, \vec{\alpha})$ are measured on a trajectory outside the support of $f(\vec{r})$. If $\vec{r}$ is on the line connecting $\vec{a}\left(\lambda_{1}\right)$ and $\vec{a}\left(\lambda_{2}\right)$, then:

$$
\begin{aligned}
& \int_{\lambda_{1}}^{\lambda_{2}} d \lambda \frac{e^{-\mu\|\vec{r}-\vec{a}(\lambda)\|}}{\|\vec{r}-\vec{a}(\lambda)\|} \frac{\partial}{\partial \lambda} p\left(\lambda, \vec{\alpha}^{*}\right)+\mu \int_{\lambda_{1}}^{\lambda_{2}} d \lambda \frac{e^{-\mu\|\vec{r}-\vec{a}(\lambda)\|}}{\|\vec{r}-\vec{a}(\lambda)\|}\left(\vec{a}^{\prime}(\lambda) \cdot \vec{\alpha}^{*}\right) p\left(\lambda, \vec{a}^{*}\right) \\
= & -2 \pi \mathrm{pv} \int_{-\infty}^{\infty} d t^{\prime} \frac{\cosh \left(\mu t^{\prime}\right)}{\pi t^{\prime}} f\left(\vec{r}-t^{\prime} \vec{e}\right),
\end{aligned}
$$

where "pv" in front of the integral indicates the principle value. The unit vector $\vec{e}$ is located along the line connecting 


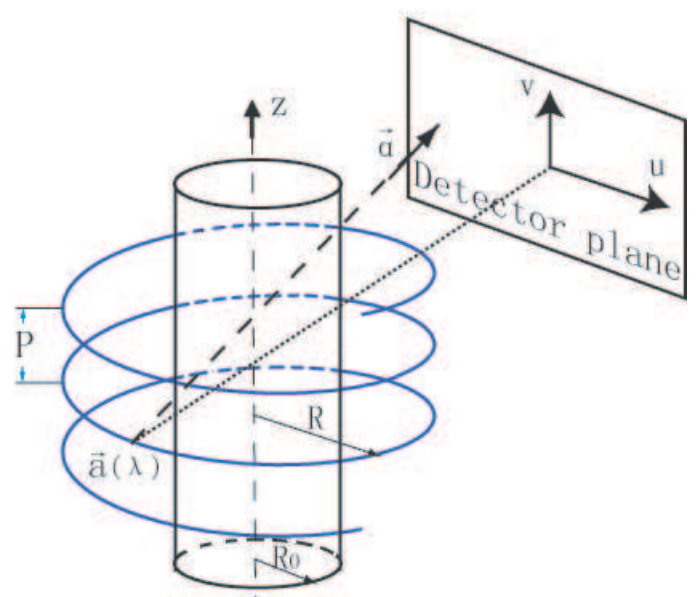

Fig. 1: Illustration of cone-beam data acquisition.

two focal points, as shown in Fig. 2. The differentiation $\frac{\partial}{\partial \lambda} p\left(\lambda, \vec{\alpha}^{*}\right)$ is performed with respect to $\lambda$ and evaluated at the direction $\vec{\alpha}^{*}$.

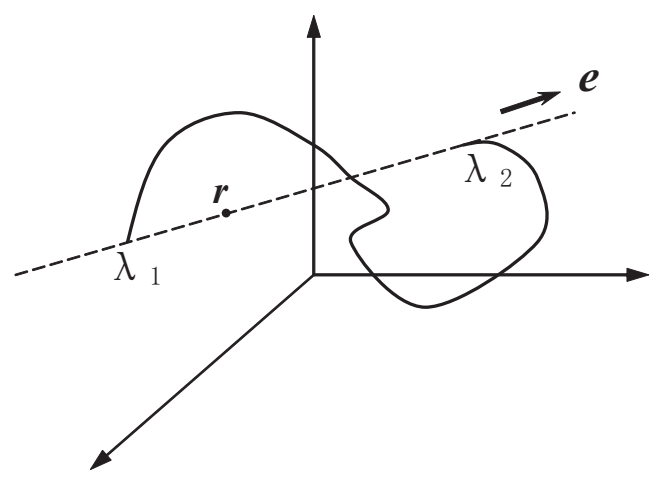

Fig. 2: The vector $\vec{r}$ is located on the line connecting $\vec{a}\left(\lambda_{1}\right)$ and $\vec{a}\left(\lambda_{2}\right)$. The trajectory of the focal point is outside the support of $f(\vec{r})$. Notations in bold in the figure indicate vectors.

The proof of the theorem is presented in Appendix I.

Theorem 1 builds a connection between projections and the true image. The DBP of projection data forms the cosh-weighted Hilbert transform of the true image along the direction determined by the two limits of the backprojection. From projection data, the left hand side of the equality is readily obtained by performing differentiation, weighting, and backprojection of the exponential ray transform. The right hand side is an image obtained by convolving the true image with a one-dimensional (1D) kernel $\frac{\cosh (\mu t)}{\pi t}$ multiplied by some factor. Thus, the image reconstruction is accomplished by inverting the convolution corresponding to a cosh-weighted Hilbert transform [18]. The following section describes how to invert the cosh-weighted Hilbert transform.

\section{EXPlicit InVERSiON OF THE FINITE HilbERT TRANSFORM}

Since the right hand side of the equality in Theorem 1 can be obtained from the projection data, the image reconstruction is reduced to inverting the cosh-weighted Hilbert transform. The inversion will be discussed as an 
independent problem in this section. After the notations and definitions are clarified, the explicit inversion formulas will be given.

The cosh-weighted Hilbert transform of $h(t)$ is defined as:

$$
H_{\mu}(s)=\frac{1}{\pi} \int_{-\infty}^{\infty} \frac{\cosh (\mu(s-t))}{s-t} h(t) d t
$$

The original function $h(t)$ is assumed to be a smooth function with compact support $t \in I_{q}=[-q, q]$. It is obvious that when $\mu=0$, the cosh-weighted Hilbert transform simplifies to the Hilbert transform [21]. However, when $\mu \neq 0$, it is more complicated since $H_{\mu}(s)$ goes to $\infty$ as $s$ goes to $\infty$, i.e., the cosh-weighted Hilbert transform is not a bounded operator. It should also be pointed out that although $h(t)$ has a compact support, the support of $H_{\mu}(s)$ can be the entire real axis $\mathbb{R}$. In SPECT, only finite data samples are available. Hence, the inversion for finite samples requires $H_{\mu}(s)$ to be known in a finite interval. This is the so-called inversion of the finite cosh-weighted Hilbert transform.

Rullgård [15] first proposed the possibility of using the inversion of the finite cosh-weighted Hilbert transform in tomography. Existence, uniqueness, and stability were shown later [17], [18]. The three papers ( [15], [17], and [18]) all developed some explicit inversion formulas. Each formula contains a function that does not have a closed-form expression. Some inversion formulae have a shift-invariant kernel, however require that data in $[-2 q, 2 q]$ for $H_{\mu}(s)$ be known [15], [18]. The others use a shift-variant kernel and demand only those data in $[-q, q]$ for $H_{\mu}(s)$, which is required for an ROI reconstruction in SPECT with truncated data [17], [18].

The following derivation is based on [18]. From [21] we know that a smooth function $h(t)$ with compact support $t \in I_{q}=[-q, q]$ can be restored from its Hilbert transform $H(s)$ in the support $s \in I_{q}$, i.e.,

$$
h(t)=\frac{\sqrt{q^{2}-t^{2}}}{\pi} \int_{-q}^{q} \frac{1}{s-t} \frac{H(s)}{\sqrt{q^{2}-s^{2}}} d s,
$$

where

$$
H(s)=\frac{1}{\pi} \int_{-\infty}^{\infty} \frac{h(t)}{s-t} d t=\frac{1}{\pi} \int_{-q}^{q} \frac{h(t)}{s-t} d t
$$

Similar to (4), a function $\hat{h}(t)$ can be constructed, but with the Hilbert transform $H(s)$ replaced with the coshweighted Hilbert transform $H_{\mu}(s)$ :

$$
\hat{h}(t)=\frac{\sqrt{q^{2}-t^{2}}}{\pi} \int_{-q}^{q} \frac{1}{s-t} \frac{H_{\mu}(s)}{\sqrt{q^{2}-s^{2}}} d s .
$$


The right hand side of (6) is manipulated by substituting in the definition (3)

$$
\begin{aligned}
& \frac{\sqrt{q^{2}-t^{2}}}{\pi} \int_{-q}^{q} \frac{1}{s-t} \frac{1}{\sqrt{q^{2}-s^{2}}}\left[\frac{1}{\pi} \int_{-\infty}^{\infty} \frac{\cosh (\mu(s-\tau))}{s-\tau} h(\tau) d \tau\right] d s \\
= & \frac{\sqrt{q^{2}-t^{2}}}{\pi} \int_{-q}^{q} \frac{1}{s-t} \frac{1}{\sqrt{q^{2}-s^{2}}}\left[\frac{1}{\pi} \int_{-\infty}^{\infty} \frac{\cosh (\mu(s-\tau))-1}{s-\tau} h(\tau) d \tau\right] d s \\
+ & \frac{\sqrt{q^{2}-t^{2}}}{\pi} \int_{-q}^{q} \frac{1}{s-t} \frac{1}{\sqrt{q^{2}-s^{2}}}\left[\frac{1}{\pi} \int_{-\infty}^{\infty} \frac{h(\tau)}{s-\tau} d \tau\right] d s .
\end{aligned}
$$

The second term is $h(t)$ according to equations (5) and (4). Introducing some notations, we have

$$
\hat{h}(t)=\int_{-q}^{q} K_{\mu}(t, p) h(p) d p+h(t)
$$

where

$$
K_{\mu}(t, p)=\sqrt{q^{2}-t^{2}} \int_{-q}^{q} \frac{A_{\mu}(\tau-p)}{\pi^{2}(\tau-t) \sqrt{q^{2}-\tau^{2}}} d \tau
$$

and

$$
A_{\mu}(t)=\frac{\cosh (\mu t)-1}{t}
$$

When $\mu=0, K_{\mu}(t, p)$ vanishes and $\hat{h}(t)=h(t)$, which is consistent with the fact that $H_{\mu}(s)=H(s)$ at $\mu=0$. When $\mu \neq 0, K_{\mu}(t, p)$ constructs a compact operator, denoted by $\Psi$. Symbolically, equation (7) becomes

$$
\hat{h}(t)=[(I+\Psi) h](t) .
$$

It was shown in [22] that if the eigenvalues of $\Psi$ are different from -1 , the operator $(I+\Psi)^{-1}$ exists, and is bounded. There is no proof that all of the eigenvalues of $\Psi$ are different from -1 for all $\mu$. However, for typical values of $\mu$ in emission tomography, the numerical study shows that $(I+\Psi)^{-1}$ exists, and is bounded. In that case, the desired function can be obtained as:

$$
h(t)=(I+\Psi)^{-1} \hat{h}(t)
$$

In an application of SPECT reconstruction, the image is restored by inverting the cosh-weighted Hilbert transform (i.e., the DBP) along the direction determined by the two limits of the backprojection.

\section{NUMERICAL STUDY}

Theorem 1 can be applied to any geometry with a smooth orbit for the focal point, as long as for each point $\vec{r}$ there is a chord that connects two focal points and passes through $\vec{r}$ in between. This chord is known as the PI-line. In this section, we present a numerical study for a helical cone-beam geometry because of its simplicity to implement mechanically given existing SPECT systems, and also due to the fact that for a helical cone-beam 
geometry an ROI can be covered by PI-lines.

\section{A. System Configuration}

The helical trajectory had a radius $R=32 \mathrm{~cm}$ and a pitch $P=4 \mathrm{~cm}$. Corresponding to each focal point $\vec{a}(\lambda)=\left(R \cos \lambda, R \sin \lambda, \frac{P}{2 \pi} \lambda\right)$, there was a detector plane consisting of a grid with $256 \times 64$ voxels of $0.78 \times 0.78$ $\mathrm{mm}^{2}$. The detector was assumed to contain the $z$-axis and was perpendicular to $(R \cos \lambda, R \sin \lambda, 0)$. In Fig. 3 the detector is shown separate from the cylinder for better visualization. Cartesian coordinates $(u, v)$ are introduced in the detector plane centered at $\left(0,0, \frac{P}{2 \pi} \lambda\right)$, where the $v$-axis is parallel to and in the same direction as $z$, and the $u$-axis is parallel to the $x-y$ plane. Note that the $(u, v)$ system is a local coordinate system, determined by the view angle $\lambda$.

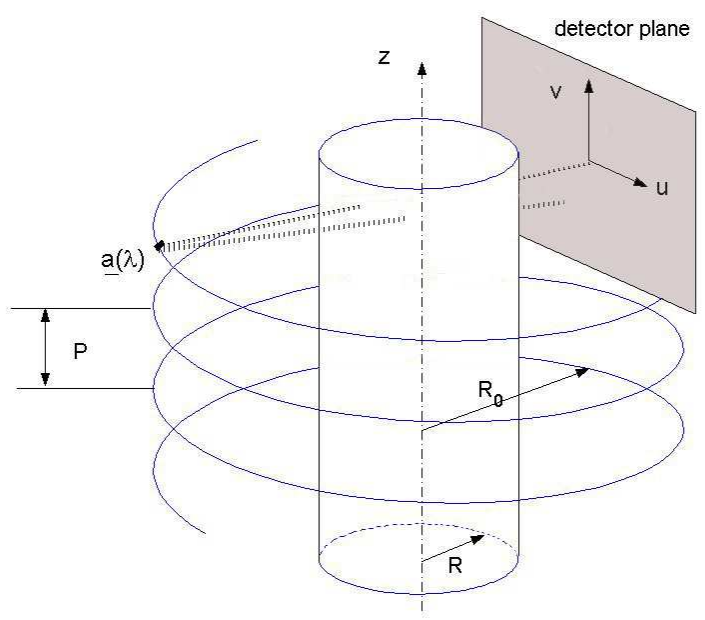

Fig. 3: Geometry of the data acquisition.

The cylinder in Fig. 3 indicates the support of the object that is scanned in SPECT, for example, the patient head in brain imaging. Since brain imaging is a clinical application that assumes a uniform attenuation coefficient, the 3D Shepp-Logan phantom, composed of the summation of the concentration of 10 ellipsoids, was chosen for the numerical study. The parameters of these ellipsoids are shown in Table I. In a global Cartesian coordinate system, each ellipsoid was centered at $\left(x_{0}, y_{0}, z_{0}\right)$, with the semi-axes of lengths $a, b$, and $c \mathrm{~cm}$; azimuthal angle $\theta$; and polar angle $\phi$. The concentration in the structure was designed by addition of the concentration in each ellipsoid. The emission contrast between internal brain structures was set to what one might experience for one particular SPECT brain scan. This contrast was much greater than what would be utilized for an $\mathrm{x}$-ray CT scan. The three central slices of the phantom are shown in Fig. 4.

The attenuation coefficient was set to be $\mu=0.15 \mathrm{~cm}^{-1}$, which is the attenuation coefficient of water at the photon energy of $140 \mathrm{keV}$.

Overall, 1600 projections for $\lambda \in[-2 \pi, 2 \pi)$ were generated. Each projection measured on the detector was 


\begin{tabular}{|c|c|c|c|c|c|c|c|c|}
\hline$x_{0}$ & $y_{0}$ & $z_{0}$ & $\mathrm{a}$ & $\mathrm{b}$ & $\mathrm{c}$ & $\theta$ & $\phi$ & density \\
\hline \hline 0 & 0 & 0 & 9.2 & 6.9 & 9.2 & 0 & 90 & 2 \\
\hline 0 & -0.184 & 0 & 8.74 & 6.624 & 8.74 & 0 & 90 & -0.8 \\
\hline 2.2 & 0 & 0 & 3.1 & 1.1 & 3.1 & 0 & 72 & -0.8 \\
\hline-2.2 & 0 & 0 & 4.1 & 1.6 & 4.1 & 0 & 108 & -0.8 \\
\hline 0 & 3.5 & 0 & 2.5 & 2.1 & 2.5 & 0 & 90 & 0.4 \\
\hline 0 & 1 & 0 & 0.46 & 0.46 & 0.46 & 0 & 0 & 0.4 \\
\hline 0 & -1 & 0 & 0.46 & 0.46 & 0.46 & 0 & 0 & 0.4 \\
\hline-0.8 & -6.05 & 0 & 0.46 & 0.23 & 0.46 & 0 & 0 & 0.4 \\
\hline 0 & -6.05 & 0 & 0.23 & 0.23 & 0.23 & 0 & 0 & 0.4 \\
\hline 0.6 & -6.05 & 0.06 & 0.46 & 0.23 & 0.46 & 0 & 90 & 0.4 \\
\hline
\end{tabular}

TABLE I: Parameters of the phantom ellipsoids. Each ellipsoid is centered at $\left(x_{0}, y_{0}, z_{0}\right)$, with semi-axes of lengths $a, b$ and $c \mathrm{~cm}$, azimuthal angle $\theta$, and polar angle $\phi$. The concentration in the phantom is obtained by addition of the concentration in each ellipsoid listed in the last column.

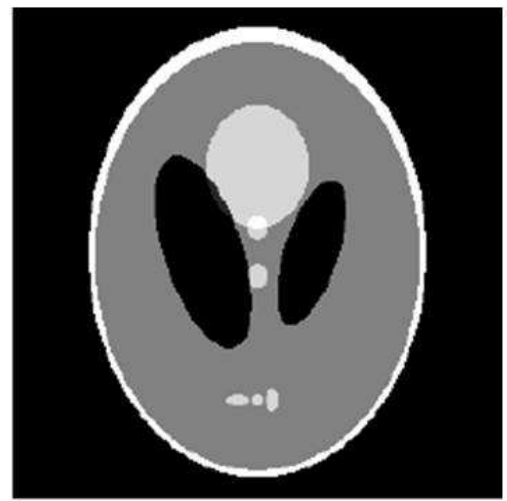

(a)

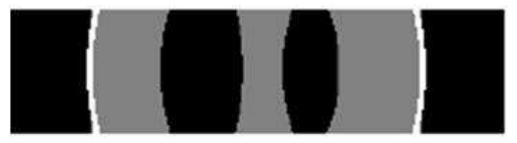

(b)

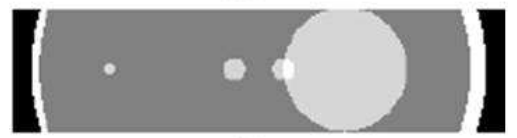

(c)

Fig. 4: Central slices of the phantom. (a) $z=0$. The horizontal axis indicates $x$-axis. The vertical axis indicates $y$-axis. (b) $y=0$. The horizontal axis indicates $x$-axis. The vertical axis indicates $z$-axis. (c) $x=0$. The horizontal axis indicates $y$-axis. The vertical axis indicates $z$-axis. Displayed grayscale window: $[0.6,1.8]$.

$p(\lambda, u, v)=\int_{0}^{\infty} d t e^{\mu t} f(\vec{a}(\lambda)+t \vec{\alpha})$ where

$$
\vec{\alpha}=\frac{1}{\sqrt{u^{2}+v^{2}+D^{2}}}\left(u \vec{e}_{u}(\lambda)+v \vec{e}_{v}(\lambda)+D \vec{e}_{w}(\lambda)\right)
$$


The three orthogonal directions are defined by

$$
\begin{aligned}
\vec{e}_{u}(\lambda) & =(-\sin \lambda, \cos \lambda, 0), \\
\vec{e}_{v}(\lambda) & =(0,0,1), \\
\vec{e}_{w}(\lambda) & =(-\cos \lambda,-\sin \lambda, 0) .
\end{aligned}
$$

As shown in Fig. 5, we denote the distance between the focal point to the detector as $D$. In our system configuration, this distance is in fact the radius of the helix: $D=R$.

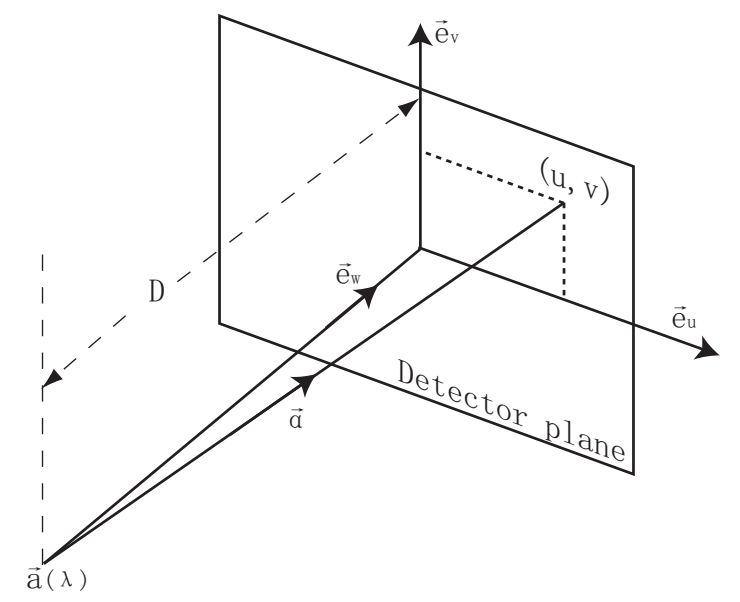

Fig. 5: Coordinate system of the data acquisition.

\section{B. Numerical Implementation}

In order to reconstruct the intensity at the point $\vec{r}$, let us first discuss the well-known PI-lines. A PI-line is defined as the line connecting two focal points $\vec{a}\left(\lambda_{1}\right)$ and $\vec{a}\left(\lambda_{2}\right)$ on the helix with $0<\lambda_{2}-\lambda_{1}<2 \pi$. It is known that for each point $\vec{r}=(x, y, z)$ inside the helix (i.e., $\left.x^{2}+y^{2}<R^{2}\right)$, there exists a unique PI-line that passes through it [23], [24]. We denote the position of the point $\vec{r}$ on its PI-line by $t$, where $0<t<1$ with $t=0$ and $t=1$ corresponding to $\vec{a}\left(\lambda_{2}\right)$ and $\vec{a}\left(\lambda_{1}\right)$, respectively. Then there is a one-to-one map between the point $\vec{r}=(x, y, z)$ and $\left(\lambda_{1}, \lambda_{2}, t\right)$ :

$$
\begin{aligned}
x & =R t \cos \lambda_{1}+R(1-t) \cos \lambda_{2}, \\
y & =R t \sin \lambda_{1}+R(1-t) \sin \lambda_{2}, \\
z & =\frac{P}{2 \pi} t \lambda_{1}+\frac{P}{2 \pi}(1-t) \lambda_{2} .
\end{aligned}
$$

With the PI-line known for the point $\vec{r}$, the reconstruction can be performed in two steps. First, the DBP is calculated for data $p(\lambda, u, v)$ measured over $\lambda=\left[\lambda_{1}, \lambda_{2}\right]$. Then the inversion of the finite cosh-weighted Hilbert transform in Section III is performed along the PI-line. 
The DBP step is implemented according to the left hand side of (2) in Theorem 1. With the configuration in Fig. 5, the differentiation in the first term is rewritten as

$$
\frac{\partial}{\partial \lambda} p(\lambda, \vec{\alpha})=\frac{\partial}{\partial \lambda} p(\lambda, u, v)=p_{1}^{\prime}+\frac{d u}{d \lambda} p_{2}^{\prime}+\frac{d v}{d \lambda} p_{3}^{\prime}
$$

The second equality makes use of the chain rule, where $p_{1}^{\prime}, p_{2}^{\prime}$, and $p_{3}^{\prime}$ are three partial derivatives of the projections, with respect to three variables. From (8), we have $u=D \frac{\vec{\alpha} \cdot \vec{e}_{u}(\lambda)}{\vec{\alpha} \cdot \vec{e}_{w}(\lambda)}$ and $v=D \frac{\vec{\alpha} \cdot \vec{e}_{v}(\lambda)}{\vec{\alpha} \cdot \vec{e} w}$. Then at a fixed $\vec{\alpha}$,

$$
\begin{aligned}
\frac{d u}{d \lambda} & =D \frac{\left(\vec{\alpha} \cdot \vec{e}_{w}(\lambda)\right)\left(\vec{\alpha} \cdot \vec{e}_{u}^{\prime}(\lambda)\right)-\left(\vec{\alpha} \cdot \vec{e}_{u}(\lambda)\right)\left(\vec{\alpha} \cdot \vec{e}_{w}^{\prime}(\lambda)\right)}{\left(\vec{\alpha} \cdot \vec{e}_{w}(\lambda)\right)^{2}} \\
& =D \frac{\left(\vec{\alpha} \cdot \vec{e}_{w}(\lambda)\right)^{2}+\left(\vec{\alpha} \cdot \vec{e}_{u}(\lambda)\right)^{2}}{\left(\vec{\alpha} \cdot \vec{e}_{w}(\lambda)\right)^{2}}=D \frac{D^{2}+u^{2}}{D^{2}}=\frac{D^{2}+u^{2}}{D} \\
\frac{d v}{d \lambda} & =D \frac{\left(\vec{\alpha} \cdot \vec{e}_{w}(\lambda)\right)\left(\vec{\alpha} \cdot \vec{e}_{v}(\lambda)\right)-\left(\vec{\alpha} \cdot \vec{e}_{v}(\lambda)\right)\left(\vec{\alpha} \cdot \vec{e}_{w}^{\prime}(\lambda)\right)}{\left(\vec{\alpha} \cdot \vec{e}_{w}(\lambda)\right)^{2}} \\
& =D \frac{0+\left(\vec{\alpha} \cdot \vec{e}_{v}(\lambda)\right)\left(\vec{\alpha} \cdot \vec{e}_{u}(\lambda)\right)}{\left(\vec{\alpha} \cdot \vec{e}_{w}(\lambda)\right)^{2}}=D \frac{v u}{D^{2}}=\frac{v u}{D}
\end{aligned}
$$

The backprojection is performed for the 512 points that constitute the central part of the PI-line passing through $\vec{r}=(x, y, z)$. In the backprojection step, the integrand valued at $\left(\lambda, u^{*}, v^{*}\right)$ is superimposed. At each fixed $\lambda$, for point $\left(x_{i}, y_{i}, z_{i}\right)$ on the PI-line, we have

$$
\begin{aligned}
u^{*} & =\frac{D}{R-x_{i} \cos \lambda-y_{i} \sin \lambda}\left(-x_{i} \sin \lambda+y_{i} \cos \lambda\right), \\
v^{*} & =\frac{D}{R-x_{i} \cos \lambda-y_{i} \sin \lambda}\left(z_{i}-\frac{P}{2 \pi} \lambda\right) .
\end{aligned}
$$

In the implementation of the finite cosh-weighted Hilbert transform step, the backprojection for the central 512 points on the PI-line is assumed to be the cosh-weighted Hilbert transform $H_{\mu}(s)$ of a 1D function $h(t)$ defined in $t \in[-1,1]$. The 1D function and its transform are both sampled at $-1+\frac{\Delta}{2},-1+\frac{\Delta \times 3}{2}, \cdots, 1-\frac{\Delta}{2}$ with $\Delta=\frac{1}{256}$. Note that the attenuation coefficient should be converted from $0.15 \mathrm{~cm}^{-1}$ to a value without dimension according to the length of this PI-line. The operator $\Psi$ in Section III can be calculated once the attenuation coefficient is decided. The contribution of the integrand at singular points is not sampled.

\section{Numerical Results}

Simulation results on three sets of PI-lines with a grayscale window [0.6, 1.8], corresponding to PI-lines starting from $\vec{a}\left(\lambda_{1}\right)$ for $\lambda_{1}=-0.92 \pi,-0.67 \pi$, and $-0.42 \pi$, respectively are shown in Fig. 6 . In each image, the vertical lines are for $\lambda_{2} \in[-0.7091 \pi, 1.2912 \pi]+\lambda_{1}$. These three images are distorted because these PI-lines form a 3D surface in the space.

The numerical results on PI-lines validate the proposed algorithm. As indicated in [6] and [18], the DBH method 

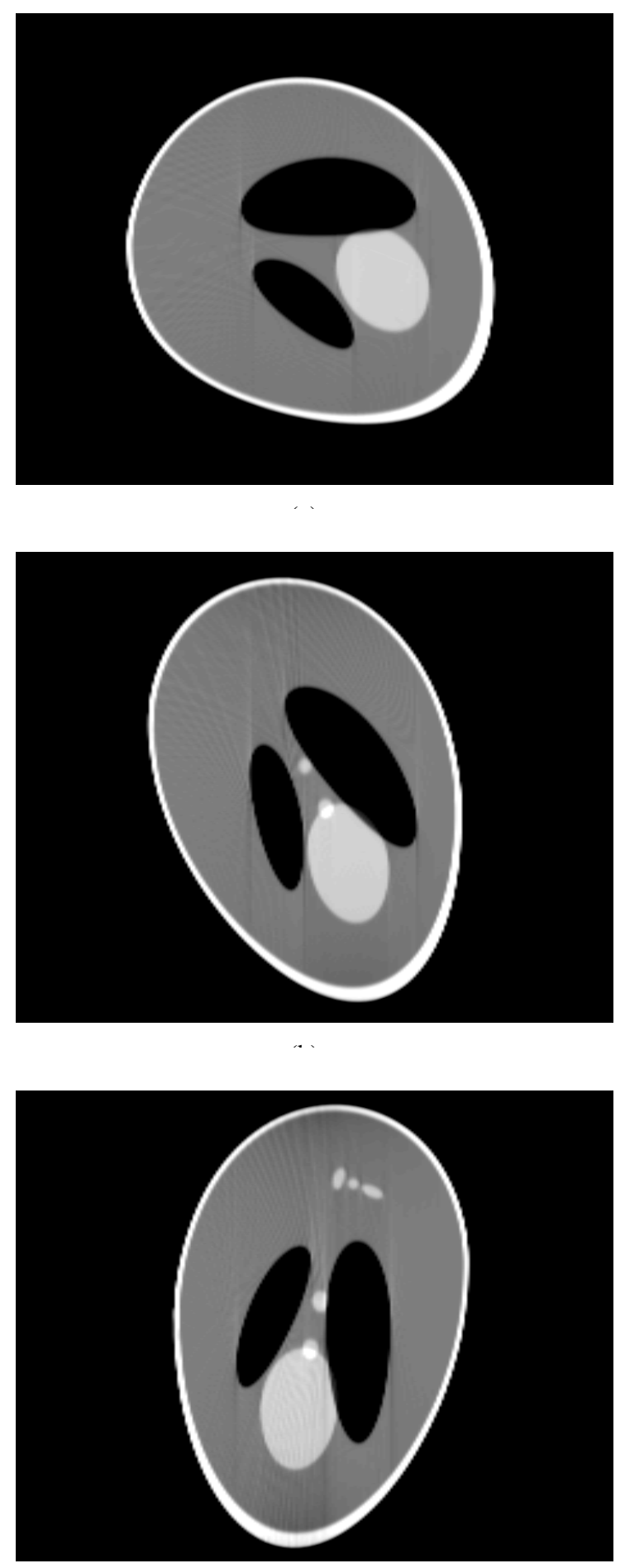

(c)

Fig. 6: Image reconstructed on PI-lines. (a) $\lambda_{1}=-0.92 \pi$. (b) $\lambda_{1}=-0.67 \pi$. (c) $\lambda_{1}=-0.42 \pi$. The horizontal axis means different $\lambda_{2} \in[0.7091 \pi, 1.2912 \pi]+\lambda_{1}$. Displayed grayscale window: [0.6,1.8].

is capable of reconstructing a particular ROI. Hence, the long object problem and the problem of truncations along the $u$-axis are both solved naturally.

To this point, the proposed 3D cone-beam algorithm consistently reconstructs objects along PI-lines accurately. The image on a Cartesian grid needs to be interpolated from the value along PI-lines. Fig. 7 displays the three 
central slices of the interpolated image. Compared to Fig. 4, these slices are applicable for quantitative studies.

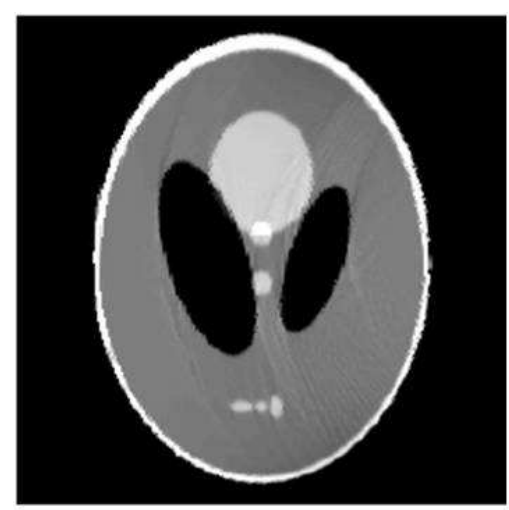

(a)

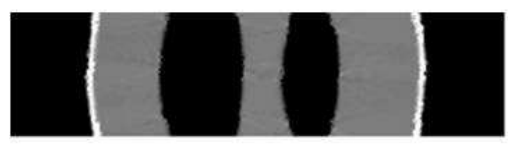

(b)

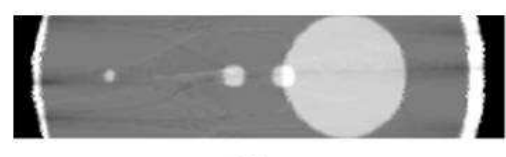

(c)

Fig. 7: Central slices of the reconstructed image. (a) $z=0$. The horizontal axis indicates $x$-axis. The vertical axis indicates $y$-axis. (b) $y=0$. The horizontal axis indicates $x$-axis. The vertical axis indicates $z$-axis. (c) $x=0$. The horizontal axis indicates $y$-axis. The vertical axis indicates $z$-axis. Displayed grayscale window: $[0.6,1.8]$.

\section{CONCLUSION}

A theoretically exact image reconstruction algorithm is presented for cone-beam SPECT with uniform attenuation compensation. The proposed algorithm has a format of differentiation, backprojection, and 1D inverse weighted Hilbert transform and thus is not as computationally demanding as an iterative method. The method also successfully solves the long object and truncation problems that result from using relatively small detectors.

For more efficient algorithms, interpolation methods suggested in [25] can be adopted to obtain the 3D image from reconstructions on PI-lines. 


\section{APPENDIX A}

\section{Proof of TheOREM 1 (SEe Also [26])}

Proof: By definition, the differentiation of the projection data with respect to the view angle in the direction $\vec{\alpha}^{*}$ is

$$
\frac{\partial}{\partial \lambda} p\left(\lambda, \vec{\alpha}^{*}\right)=\int_{0}^{\infty} d t e^{\mu t} \vec{a}^{\prime}(\lambda) \cdot(\vec{\nabla} f)\left(\vec{a}(\lambda)+t \frac{\vec{r}-\vec{a}(\lambda)}{\|\vec{r}-\vec{a}(\lambda)\|}\right)
$$

Taking the convex property of $f(\vec{r})$, the integral in the above expression is the same if the lower limit is $-\infty$. Changing the variable $\tau=1-\frac{t}{\|\vec{r}-\vec{a}(\lambda)\|}$, the differentiation becomes

$$
\begin{aligned}
& \int_{-\infty}^{\infty} d t e^{\mu t} \vec{a}^{\prime}(\lambda) \cdot(\vec{\nabla} f)\left(\left[1-\frac{t}{\|\vec{r}-\vec{a}(\lambda)\|}\right] \vec{a}(\lambda)+\frac{t}{\|\vec{r}-\vec{a}(\lambda)\|} \vec{r}\right) \\
= & \int_{-\infty}^{\infty} d \tau\|\vec{r}-\vec{a}(\lambda)\| e^{-\mu \tau\|\vec{r}-\vec{a}(\lambda)\|} e^{\mu\|\vec{r}-\vec{a}(\lambda)\|} \vec{a}^{\prime}(\lambda) \cdot(\vec{\nabla} f)(\tau \vec{a}(\lambda)+(1-\tau) \vec{r}) .
\end{aligned}
$$

Using the following equality

$$
\vec{a}^{\prime}(\lambda) \cdot(\vec{\nabla} f)(\tau \vec{a}(\lambda)+(1-\tau) \vec{r})=\frac{1}{\tau} \frac{d}{d \lambda} f(\tau \vec{a}(\lambda)+(1-\tau) \vec{r})
$$

we have

$$
\begin{aligned}
& \int_{\lambda_{1}}^{\lambda_{2}} d \lambda \frac{e^{-\mu\|\vec{r}-\vec{a}(\lambda)\|}}{\|\vec{r}-\vec{a}(\lambda)\|} \frac{\partial}{\partial \lambda} p\left(\lambda, \vec{\alpha}^{*}\right) \\
= & \int_{\lambda_{1}}^{\lambda_{2}} d \lambda \mathrm{pv} \int_{-\infty}^{\infty} \frac{d \tau}{\tau} e^{-\mu \tau\|\vec{r}-\vec{a}(\lambda)\|} \frac{d}{d \lambda} f(\tau \vec{a}(\lambda)+(1-\tau) \vec{r}) \\
= & \operatorname{pv} \int_{-\infty}^{\infty} \frac{d \tau}{\tau} \int_{\lambda_{1}}^{\lambda_{2}} d \lambda e^{-\mu \tau\|\vec{r}-\vec{a}(\lambda)\|} \frac{d}{d \lambda} f(\tau \vec{a}(\lambda)+(1-\tau) \vec{r}) \\
= & \left.\operatorname{pv} \int_{-\infty}^{\infty} \frac{d \tau}{\tau}\left[e^{-\mu \tau\|\vec{r}-\vec{a}(\lambda)\|} f(\tau \vec{a}(\lambda)+(1-\tau) \vec{r})\right]\right|_{\lambda_{1}} ^{\lambda_{2}} \\
- & \operatorname{pv} \int_{-\infty}^{\infty} \frac{d \tau}{\tau} \int_{\lambda_{1}}^{\lambda_{2}} d \lambda f(\tau \vec{a}(\lambda)+(1-\tau) \vec{r}) e^{-\mu \tau\|\vec{r}-\vec{a}(\lambda)\|} \\
& \times(-\mu \tau) \frac{d}{d \lambda}\|\vec{r}-\vec{a}(\lambda)\| .
\end{aligned}
$$

With $\tau=\frac{t^{\prime}}{\|\vec{r}-\vec{a}(\lambda)\|}$, the first term is simplified to

$$
\begin{aligned}
& \left.\mathrm{pv} \int_{-\infty}^{\infty} \frac{d t^{\prime}}{t^{\prime}} e^{-\mu t^{\prime}} f\left(\vec{r}-t^{\prime} \vec{\alpha}^{*}\right)\right|_{\lambda_{1}} ^{\lambda_{2}} \\
= & \mathrm{pv} \int_{-\infty}^{\infty} \frac{d t^{\prime}}{t^{\prime}} e^{-\mu t^{\prime}}\left[f\left(\vec{r}+t^{\prime} \vec{e}\right)-f\left(\vec{r}-t^{\prime} \vec{e}\right)\right] \\
= & \operatorname{pv} \int_{-\infty}^{\infty} \frac{d t^{\prime}}{t^{\prime}} \cosh \left(\mu t^{\prime}\right) f\left(\vec{r}+t^{\prime} \vec{e}\right) \\
= & -2 \pi \mathrm{pv} \int_{-\infty}^{\infty} d t^{\prime} \frac{\cosh \left(\mu t^{\prime}\right)}{\pi t^{\prime}} f\left(\vec{r}-t^{\prime} \vec{e}\right) .
\end{aligned}
$$


In the last part of (14),

$$
\begin{aligned}
& \frac{d}{d \lambda}\|\vec{r}-\vec{a}(\lambda)\| \\
= & \frac{d}{d \lambda} \sqrt{(\vec{r}-\vec{a}(\lambda)) \cdot(\vec{r}-\vec{a}(\lambda))} \\
= & \frac{-\vec{a}^{\prime}(\lambda) \cdot(\vec{r}-\vec{a}(\lambda))+(\vec{r}-\vec{a}(\lambda)) \cdot\left(-\vec{a}^{\prime}(\lambda)\right)}{2 \sqrt{(\vec{r}-\vec{a}(\lambda)) \cdot(\vec{r}-\vec{a}(\lambda))}} \\
= & \frac{-2 \vec{a}^{\prime} \cdot(\vec{r}-\vec{a}(\lambda))}{2\|\vec{r}-\vec{a}(\lambda)\|}=-\vec{a}^{\prime}(\lambda) \cdot \frac{\vec{r}-\vec{a}(\lambda)}{\|\vec{r}-\vec{a}(\lambda)\|} \\
= & -\vec{a}^{\prime}(\lambda) \cdot \vec{\alpha}^{*} .
\end{aligned}
$$

With the above equality and the change of variable $\tau=1-\frac{t^{\prime}}{\|\vec{r}-\vec{a}(\lambda)\|}$, the second term of (14) becomes

$$
\begin{aligned}
& -\mu \int_{\lambda_{1}}^{\lambda_{2}} d \lambda \int_{-\infty}^{\infty} d t^{\prime} \frac{e^{-\mu\|\vec{r}-\vec{a}(\lambda)\|}}{\|\vec{r}-\vec{a}(\lambda)\|} f\left(\vec{r}+t^{\prime} \vec{\alpha}^{*}\right) e^{\mu t^{\prime}} \vec{a}^{\prime}(\lambda) \cdot \vec{\alpha}^{*} \\
& =-\mu \int_{\lambda_{1}}^{\lambda_{2}} d \lambda \frac{e^{-\mu\|\vec{r}-\vec{a}(\lambda)\|}}{\|\vec{r}-\vec{a}(\lambda)\|} \vec{a}^{\prime}(\lambda) \cdot \vec{\alpha}^{*} p\left(\lambda, \vec{a}^{*}\right) .
\end{aligned}
$$

From (14), (15), and (16), the theorem is proved.

\section{ACKNOWLEDGMENT}

This work was supported in part by the National Institutes of Health under Grant R01 EB00121 and in part by the Director, Office of Science, Office of Biological and Environmental Research, Medical Sciences Division of the U.S. Department of Energy under Contract DE-AC02-05CH11231.

\section{REFERENCES}

[1] L. A. Feldkamp, L. C. Davis, and J. K. Kress, "Practical cone-beam algorithm,"Journal of the Optical Society of America A., vol. 1, pp. 612-619, 1984.

[2] H. K. Tuy, “An inversion formula for cone-beam reconstruction,” SIAM J Appl. Math., vol. 43, pp. 546-552, 1983.

[3] B. D. Smith, "Image reconstruction from cone-beam projections: necessary and sufficient conditions and reconstruction methods," IEEE Trans. Med. Imag., vol. MI-4, pp. 14-28, 1985.

[4] P. Grangeat, "Mathematical framework of cone beam 3D reconstruction via the first derivative of the Radon transform," Mathematical methods in Tomography, vol. 1497, pp. 66-97, 1991.

[5] A. Katsevich, "Analysis of an exact inversion algorithm for spiral cone-beam CT," Phys. Med. Biol., vol. 47, pp. 2583-2597, 2002.

[6] Y. Zou and X. Pan, "Exact image reconstruction on PI-lines from minimum data in helical cone-beam CT," Phys. Med. Biol., vol. 49, pp. 941-959, 2004.

[7] G. L. Zeng, G. T. Gullberg, B. M. W. Tsui, and J. A. Terry, "Three-dimensional iterative reconstruction algorithms with attenuation and geometric point response correction,” IEEE Trans. Nucl. Sci., vol. 38, pp. 693-702, 1991.

[8] A. Welch, S. Webb, and M. Flower, "Improved cone-beam SPECT via an accurate correction for non-uniform photon attenuation," IEEE Trans. on Nucl. Sci., vol. 38, pp. 909-928, 1993. 
[9] D. R. Gilland, R. J. Jaszczak, H. Wang, T. G. Turkington, K. L. Greer, and R. E. Coleman, “A 3D model of non-uniform attenuation and detector response for efficient iterative reconstruction in SPECT," Phys. Med. Bio., vol. 39, pp. 547-561, 1994.

[10] V. La and P. Grangeat, "Minimal residual cone-beam reconstruction with attenuation correction in SPECT," Phys. Med. Bio., vol. 43, pp. 715-727, 1998.

[11] V. P. Palamodov, “An inversion method for an attenuated X-ray transform," Inverse Problems, vol. 12 pp. 717-729, 1996.

[12] J. Li, Z. Liang, J. Ye, and G. Han, "Implementation and preliminary investigation of analytical methods for correction of distancedependent resolution variation and uniform attenuation in 3D brain SPECT," IEEE Trans. Nucl. Sci., vol. 46, pp. $2162-2171,1999$.

[13] J. M. Wagner and F. Noo, “Three-dimensional image reconstruction from exponential parallel-beam projections," IEEE Trans. on Nucl. Sci., vol. 48, pp. 743-749, 2001.

[14] L. A. Kunyansky, "Inversion of the 3D exponential parallel-beam transform and the Radon transform with angle-dependent attenuation," Inverse Problems, vol. 20, pp. 1455-1478, 2004.

[15] H. Rullgård, “An explicit inversion formula for the exponential Radon transform using data from 180 degrees," Ark. Math., vol. 42, pp. 353-362, 2004

[16] Q. Huang, G. L. Zeng, and G. T. Gullberg, "An analytical inversion of the $180^{\circ}$ exponential Radon transform with a numerically generated kernel," International Journal of Image and Graphics, vol. 7, pp. 71-85, 2007.

[17] F. Noo, M. Defrise, J. Pack, and R. Clackdoyle, "Image reconstruction from truncated data in SPECT with uniform attenuation," Inverse Problems, vol. 23, pp. 645-668, 2007.

[18] J. You, G. L. Zeng, and Q. Huang, "Finite Inversion of the Weighted Hilbert Transform - Application to medical imaging," Proceedings of Meeting on Fully 3D Image Reconstruction in Radiology and Nuclear Medicine, Lindau, Germany, pp. 233-236, 2007.

[19] Q. Huang, J. You, G. L. Zeng, and G. T. Gullberg, "Reconstruction from uniformly attenuated SPECT projection data using the DBH method," IEEE Trans. Med. Imag., in press, 2008.

[20] Z. Liang, J. Ye, and D. P. Harrington, "Quantitative brain SPECT in three dimensions: an analytical approach without transmission scans," Three-Dimensional Image Reconstruction in Radiology and Nuclear Medicine, Kluwer Academic Publishers, pp. 117-132, 1996.

[21] F. G. Tricomi, Integral Equations, Interscience Publisher Inc, New York, 1957.

[22] W. Rudin, Functional Analysis, McGraw-Hill Book Company, New York, 1973.

[23] P. E. Danielsson, P. Edholm, J. Eriksson, and M. Magnusson, "Towards exact reconstruction for helical cone-beam scanning of long objects. A new detector arrangement and a new completeness condition,” Proceedings of Meeting on Fully 3D Image Reconstruction in Radiology and Nuclear Medicine, (Pittsburgh, Pa) ed. D. W. Townsend and P. E. Kinahan, pp. 141-144, 1997.

[24] M. Defrise, F. Noo, and H. Kudo, "A solution to the long-object problem in helical cone-beam tomography," Phys. Med. Biol., vol. 45, pp. 623-643, 2000.

[25] S. Žabić, S. Hoppe, F. Dennerlein, G. Lauritsch, and F. Noo, "Resampling density values on R-lines into density values on a Cartesian grid," Nuclear Science Symposium Conference Record, vol. 4, pp. 2299-2303, 2006.

[26] F. Noo and J. D. Pack, "Theory for image reconstruction from divergent-beam projections in SPECT," IEEE Nuclear Science Symposium Conference Record, vol. 6, pp. 3449-3452, 2006. 